M exico, there is an additional twist to the differentiation of gender roles, in the sense that women are expected not to drink alcohol, because it is not compatible with their domestic responsibilities. And so we come back to the issue of domestic violence which was raised by Clark and Wyshak, with the misuse of alcohol by men being closely correlated with physical violence against female partners.

Psychiatrists around the world need to be alert to the continuing stresses on w omen, from family, partners, work and many other obligations; cultural influences on female mental health are rarely trivial.

\title{
The confluence of violence towards an intimate partner, substance misuse and mental health: a worldwide problem affecting women
}

\author{
Cari Jo Clark, MPH ScD ${ }^{1}$ and Grace Wyshak, $\mathrm{PhD}^{2}$ \\ ${ }^{1}$ Department of Population and International Health, Harvard School of Public Health, Boston, Massachusetts, \\ USA \\ ${ }^{2}$ Associate Professor, Department of Psychiatry, Harvard Medical School, and Departments of Biostatistics \\ and Population and International Health, Harvard School of Public Health, 665 Huntington Avenue, Boston, \\ MA 02115, USA, email wyshak@hsph.harvard.edu
}

$V$ iolence towards an intimate partner, substance misuse and other mental health disorders are problems that tend to cluster together and result in multiple burdens for afflicted individuals (Desjarlais et al, 1995; Wyshak \& Modest, 1996; Wyshak, 2000). They are prevalent not only in high-risk groups but also among members of the general public seeking primary healthcare (Bauer et al, 2000; World Health O rganization, 2001), where their afflictions often go undiagnosed and untreated (Edlund et al, 2004; Kramer et al, 2004). Furthermore, violence towards an intimate partner, substance misuse and other mental health disorders involve common symptom pathways, such as psychiatric distress, headache, abdominal pain, gastrointestinal problems and multiple somatic complaints (Berwick et al, 1991), which suggests that the use of an integrated set of screening instruments may lead to early detection and treatment for patients who are suffering from one or more of these problems.

\section{The role of gender}

Violence directed at an intimate partner disproportionately affects women: between $10 \%$ and $69 \%$ of women have at some time experienced such violence, according to a review of 48 population-based studies from a variety of countries around the world (Krug et al, 2002). Similarly important, tobacco smoking, alcohol consumption and the use of illicit drugs (excluding ecstasy, solvents and cannabis) cause a total of approximately 6.9 million deaths annually: 4.9, 1.8 and 0.2 million, respectively (World Health 0 rganization, 2002). Although men use these substances at higher rates than women, the health impact for women is no less significant and for some substances may be even greater (Cormier et al, 2004). Furthermore, neuropsychiatric disorders as a whole afflict approximately 450 million individuals worldwide (World Health $O$ rganization, 2001). Of these disorders, depression, which disproportionately affects women, is the leading cause of disability worldwide (World $H$ ealth 0 rganization, 2001).

\section{Confluence of the three types of problem}

Violence towards an intimate partner, substance misuse and other mental health diso rders are highly correlated with each other. Substance misuse and other mental health disorders are risk factors for and health outcomes of violence perpetrated by an intimate partner (Riggs et al, 2000). Substance misuse and other mental disorders themselves are highly correlated under the rubric of dual diagnosis. Research specifically addressing these interconnections has found high correlations between the experience of violence, substance misuse and psychiatric distress, characterised by gender and ethnic differences; notably, in women depression and alcoholism are highly correlated (W yshak \& Modest, 1996).
Violence directed at an intimate partner disproportionately affects women: between $10 \%$ and $69 \%$ of women have at some time experienced such violence, according to a

review of 48 population-based

studies from a variety of

countries around the world. 


\section{Screening}

Wyshak \& Modest (1996) have demonstrated the feasibility of screening for these multiple but interrelated problems. Screening efforts for alcohol, drug and mental disorders have increased over time, but recent survey research of primary-care patients in the U nited States indicates that such screening is generally still lacking. For example, in one survey approximately half of those who reported substance misuse or mental disorder were screened; even among those screened and found to have a disorder, $40 \%$ received no treatment (Edlund et al, 2004). Even fewer female respondents (25\%) reported being screened for violence in another sample of primary-care patients, although $83 \%$ supported the idea (Kramer et al, 2004).

These findings suggest that while screening efforts continue to proliferate, they remain under-used tools for early detection and treatment. Screening instruments that address these interrelated conditions - violence, substance misuse and mental disorders - may lead to improved identification and treatment of afflicted individuals. Moving beyond recognition of vio lence directed at an intimate partner requires the development and examination of culturally relevant interventions.

\section{Intervention and the role of culture}

Effective interventions in mental health and substance misuse exist (World H ealth 0 rganization, 2001) but the effectiveness of those that seek to reduce violent incidents remains understudied (W athen $\&$ MacMillan, 2003), which presents a barrier to the provision of ethical, safe, evidence-based interventions for women. There is a further problem, in that these interventions have been designed in and for the populations of developed countries: they therefore require context-specific adaptation for their use elsewhere in the world, for different populations. Such adaptation will need to take into consideration the obstacles and resources available, including referral sources in the case of violence towards an intimate partner (G arcia-M oreno, 2002).

Cultural contexts underpin the feasibility and acceptability of any healthcare interventions, but this is especially true where attitudes to violence against women (including victim blaming) as well as the stigma attached to mental illness and substance misuse are to the fore. Therefore, context-specific programming and evaluation are necessary. This will prove difficult given the resource constraints that violence, substance misuse and mental health programmes face worldwide, but especially so in low-income countries.

\section{Conclusions}

The confluence and common symptom pathways of violence, substance misuse and other mental health disorders call for integrated screening (Wyshak \& Modest, 1996) and treatment interventions for women. This will require training for healthcare providers, to enable them to recognise and treat these interrelated problems, and to deal with the associated ethical issues, such as privacy and confidentiality (Wyshak, 2000). Research and funding are required to develop culturally appropriate interventions and to evaluate their effectiveness (Garcia-Moreno, 2002). These efforts are necessary to ensure that all those women, worldwide, who face these problems receive effective, culturally appropriate health interventions in the primary-care setting.

\section{References}

Bauer, H. M., Rodriguez, M. A. \& Perez-Stable, E. J. (2000) Prevalence and determinants of intimate partner abuse among public hospital primary care patients. Journal of General Internal Medicine, 15, 811-817.

Berwick, D. M., Murphy, J. M., Goldman, P. A., et al (1991) Performance of a five-item mental health screening test. Medical Care, 29, 169-176.

Cormier, R. A., Dell, C. A. \& Poole, N. (2004) Women and substance abuse problems. BMC Women's Health, 4 (suppl. 1), S8.

Desjarlais, R., Eisenberg, L., Good, B., et al (1995) World Mental Health: Problems and Priorities in Low-Income Countries. N ew York: Oxford University Press.

Edlund, M. J., Unutzer, J. \& Wells, K. B. (2004) Clinician screening and treatment of alcohol, drug, and mental problems in primary care: results from healthcare for communities. Medical Care, 42, 1158-1166.

Garcia-Moreno, C. (2002) Dilemmas and opportunities for an appropriate health-service response to violence against women. Lancet, 359, 1509-1514.

Kramer, A., Lorenzon, D. \& Mueller, G. (2004) Prevalence of intimate partner violence and health implications for women using emergency departments and primary care clinics. Women's Health Issues, 14, 19-29.

Krug, E. G., Dahlberg, L. L., Mercy, J. A., et al (eds) (2002) World Report on Violence and Health. Geneva: WHO.

Riggs, D. S., Caulfield, M. B. \& Street, A. E. (2000) Risk for domestic violence: factors associated with perpetration and victimization. Journal of Clinical Psychology, 56, 1289-1316.

Wathen, C. N . \& MacMillan, H. L. (2003) Interventions for violence against women: scientific review. Journal of the American Medical Association, 289, 589-600.

World Health O rganization (2001) The World Health Report, 2001. Mental Health: New Understanding, New Hope. Geneva: WHO

World Health O rganization (2002) The World Health Report, 2002: Reducing Risks, Promoting Healthy Life. Geneva: WHO.

Wyshak, G. (2000) Violence, mental health, substance abuse - problems for women worldwide. Health Care for Women International, 21, 631-639.

Wyshak, G. \& Modest, G. A. (1996) Violence, mental health, and substance abuse in patients who are seen in primary care settings. Archives of Family Medicine, 5, 441-447. 